COMMENT. Migraine is a rare precipitant of acute ischemic stroke, mostly occurring in young women in association with attacks of migraine with aura (see Progress in Pediatric Neurology III, PNB Publishers, 1997;174-5). Triptans constrict coronary arteries, and triptan-induced chest pain may mimic angina. Triptans are contraindicated in patients with ischemic heart disease, and some uncontrolled reports of triptan-associated stroke have limited their use in some cases of migraine. The above controlled study has shown that in general practice, triptan treatment in migraine does not increase the risk of stroke, as long as the treatment is used in patients less at risk of stroke. A slightly higher risk of stroke in the non-triptan-treated group with migraine is noteworthy. The safety and efficacy of triptans has not been established for use in children (PDR 2003).

\title{
HEADACHE WITH BENIGN ALTERNATING HEMIPLEGIA
}

An 8-year-old boy with benign familial nocturnal alternating hemiplegia of childhood (BNAHC) and severe headache and vomiting during episodes is reported from the University of Rochester, NY. Episodes of nocturnal hemiparesis began at 3 years of age, and they occur several times a week to once a month. They are complicated by dystonic posturing of hands and feet, and they last 20 minutes to 5 hours (average, 2 to 3 hours). Headache that begins after 20 minutes following onset of hemiparesis lasts up to 7 hours, and is followed by fatigue. Triggers include physical and emotional stress and sleep deprivation, while sleep alleviates the symptoms. Medications (flunarizine, phenytoin, valproate, carbamazepine, and ibuprofen) and elimination of migraine dietary triggers were ineffective. EEGs showed contralateral hemispheric slowing during or after an attack. Type 1 diabetes with one generalized hypoglycemic seizure, and hyperactive and oppositional behavior with symptoms of depression and threatened suicide developed later. The family history was positive for migraine (in the mother and father), seizures (great-grandmother), and depression and bipolar disease, but negative for hemiparesis or dystonia. The authors tabulate the clinical characteristics of 6 patients with BNAHC previously reported, 6 having episodes of flaccid hemiparesis, 1 also dystonic and ataxic, 5 having a family history of migraine, and 3 being hyperactive (Andermann E et al. 1994; Chaves-Vischer et al. 2001). (Kavanaugh M, Myers GJ. Benign alternating hemiplegia of childhood: new features and associations. Neurology February (2 of 2) 2004;62:672). (Reprints: Dr Gary J Myers, University of Rochester Medical Center, 601 Elmwood Ave, Box 631, Rochester, NY 14642).

COMMENT. Severe headache and vomiting during episodes were described as unique features in this patient with benign alternating hemiplegia of childhood (BNAHC). The initial report of this syndrome by Andermann E et al. 1994 (see Progress in Pediatric Neurology III, PNB Publ, 1997;171-2) described two brothers with recurrent attacks arising out of sleep, distinguishable from the classic, sporadic form of $\mathrm{AHC}$ having a poor prognosis. Both infants with BNAHC awoke screaming $1 \frac{1}{2} \mathrm{hrs}$ after falling asleep and were paralyzed on one side. After returning to sleep and awakening in the morning, they had recovered. Similar episodes recurred with increasing frequency. Features such as hypotonia, dystonia, and eye movements, characteristic of AHC, were absent. Development remained normal. Both parents suffered from migraine. The etiology of BNAHC is unknown, but it is usually classified as a channelopathy and migraine variant. 